\title{
A New Channel Borrowing Database Algorithm in Multimedia LEO Satellite Communications
}

\author{
Debabrata Sarddar \\ (Astt Professor) \\ Department of C.S.E. \\ University of kalyani \\ Suman Kumar Sikdar \\ M. Tech in C.S.E \\ Jadavpur University
}

\author{
Soumya Das \\ Department of E.T.C.E. \\ Bengal Institute of tec.WBUT
}
Sougata Chakraborty
M.Tech in C.S.E
Jadavpur University

\author{
Dipsikha Ganguly \\ Department of E.T.C.E \\ B.P.Poddar I.M.T.,WBUT
}

\author{
Kunal Hui \\ M. Tech in C.S.E \\ Haldia Institute of Technology
}

\author{
Sujoy Palit \\ M. Tech in C.S.E \\ W.B.U.T
}

\begin{abstract}
LEO satellite networks are going to play an indispensable role in the development of ubiquitous broadband multimedia systems because of their some attracting characteristics such as low power dissipation, low propagation delay and more efficient spectrum allocation due to frequency reuse between satellites and spotbeams. So they are considered to be the replacement of terrestrial wireless networks. But as the speed of LEO satellites is high relative to the terrestrial mobile networks which moves at lower speed but at more random directions. As a result the number of handover occurrence is very frequent in LEO networks. To overcome these problems a suitable bandwidth allocation strategy along with connection admission control technique must have to be followed. Scientists have proposed many strategies but none can fulfil all the requirements. Here we have proposed a new channel borrowing database algorithm for the channel borrowing strategy. Simulation results shows that our proposed method has less call dropping probability and a better fairness index.
\end{abstract}

Keywords: Handover latency, Spot beams, LEO, Mobile Node (MN), Broadband, Multimedia Systems

\section{INTRODUCTION}

Terrestrial wireless networks such as current cellular and Personal Communication Services (PCS) systems provide mobile communication services with limited geographic coverage. To provide global coverage to a diverse user population, a number of Low Earth Orbit (LEO) satellite networks have been proposed. The LEO satellite networks can support both the areas with terrestrial wireline and wireless networks and the areas that lack any network infrastructure. In the former case, the satellite system could interact with the terrestrial wireless network to absorb the instantaneous traffic overload of these networks. In other words, mobile users could alternatively access either a terrestrial or a satellite network through dual-mode handheld terminals. In the latter application area, LEO satellites would cover regions where terrestrial wireline and wireless systems are economically infeasible because of rough terrain or insufficient user population.[1]
First generation satellite networks utilized Geostationary Earth Orbit (GEO) satellites, which are located over the equator at an altitude of $35,786 \mathrm{~km}$. At this altitude, a satellite circulates the earth in synchrony with the earth, i.e., a GEO satellite completes one turn around the earth approximately in 24 hours. As a result, the position of a GEO satellite is stationary with respect to a fixed observer on the earth surface. This is a good feature since the coverage area of a GEO satellite is also stationary. Moreover, a GEO satellite covers almost $1=3$ of the earth surface excluding the polar regions. Hence, three satellites are sufficient for global coverage. Large and stationary coverage area results from very high orbit altitude, which also results in certain disadvantages for mobile communications. First, the user terminals and the satellites have high power consumption for the communication. Second, the propagation delay between the mobile user and the satellite is too high for real-time multimedia communications. Third, high orbit altitude results in an inefficient use of the available frequency resources. [2]

An alternative to GEO satellite systems is to utilize low earth orbit satellite systems. The major advantages of these new systems are low propagation delay, low power requirements in the user terminals and the satellites, simple user terminals, and efficient spectrum utilization using small coverage area for each satellite. Moreover, it is possible to route a connection using Inter-satellite Links (ISL) without relying on terrestrial resources. However, in contrast to GEO systems, a number of mobility management problems occur in the LEO satellite systems. Mobility management in LEO satellite networks can be classified into: location management (registration and paging), and handover management.[3]

Location management tracks and locates the user terminals for the incoming calls, while handover management allows a call in progress to continue without any disruption as the serving cell of the user is changing. Location management protocols deal with querying and storing information in location databases (registration) and sending paging signals to locate the user within the network (paging). As a result, many of the issues are not protocol dependent and can be applied to any of the mobile networks, i.e., similar algorithms can be used in terrestrial wireless networks and satellite networks. In contrast, handover algorithms in the satellite networks differ 
from those in the terrestrial wireless networks. This is because the handovers occur as a result of the satellite movement as explained in the following sections. In the terrestrial wireless networks, the handovers occur because of the user movement. Hence, there is a need for further research in the satellite handover management. [4]

In this paper we have proposed a new channel borrowing database algorithm (CBDA) to improve Quality of service (QoS), less call dropping probability and have a better fairness index.

The paper is organised as follow: in the second section we have described the related channel allocation strategies available. In the third section we have described the details of CBDA strategy. In the forth section the simulation results of related channel allocation methods and CBDA strategy based ())in y. In the next section we conclude the whole paper and finally a future work is mention regarding this paper in section six.

\section{RELATED WORK}

\subsection{LEO satellite mobility model}

In this article, we assume a one-dimensional mobility model where the end users move in straight lines and at constant speed Vtrk [8], which is relevant to the satellite's altitude. Although each spot beam is circular, we model it as a rectangular bounded cell, and all the spot beams have the same shape and size. This assumption has some distortion for the real LEO mobility model, especially considering the boundary of the spot beam. However, it is acceptable to allocate channel for the spot beam from the system's view. At the considered time, the user is located at the centre of the area covered by the satellite $S 1$. When satellite $S 1$ in Fig. 1 moves toward the left, the user passes from one spot beam to the next on the right. Within the footprint of satellite $S 1$, this is a beam handover. Eventually, the user will enter the overlap area between satellites $S 1$ and $S 2$, and will experience a satellite handover. The proposed handoff strategy mainly focuses on the spot beam handover and is also suitable for the satellite handover. For convenience, we use the term "cell" as the reference to the spot beam.[5]

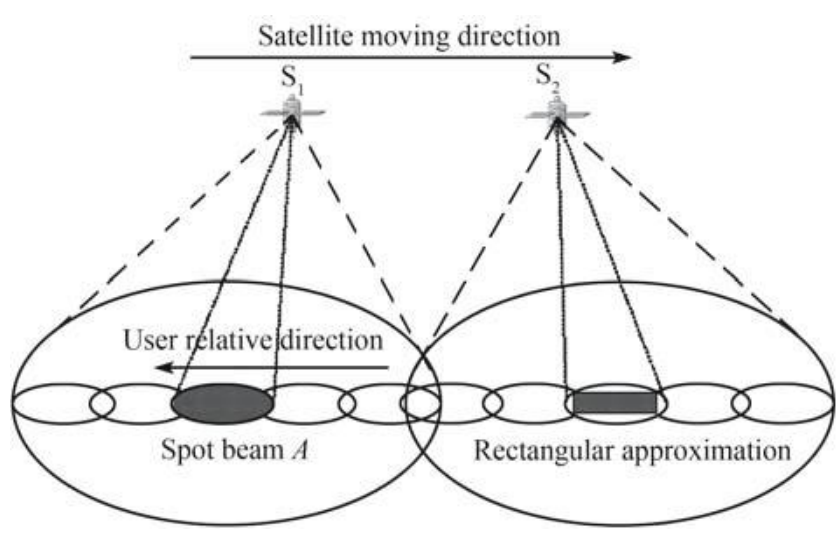

Fig. 1 LEO satellite spot beam coverage

From the outset of a call in a cell, the user travels a distance that can be assumed as uniformly distributed between 0 and $2 R$. If the cell is the "source cell" for the call, where $R$ is a constant value equal to half of the constant length of a spot beam and when a handover occurs, the user will enter a neighbouring spot beam called "transit cell" whose length is deterministically equal to $2 R$. In our model, we only consider the handover due to satellite movement, and take into no other factors into account, such as the shadowing and fading, etc. To describe both the user and LEO satellite mobility model conveniently, we assume the user's traffic duration is $T \mathrm{~m}$ and define the user's mobility parameter [8] a

$$
\mathrm{a}=2 R / V_{t r k} \cdot \mathrm{T}_{\mathrm{m}}
$$

It can be seen that the mobile station's (MS) mobility decreases if $V_{t r k}$ decreases and/or $R$ increases (i.e., a increases). Especially when $V_{t r k}$ is zero, then $\alpha$ becomes an infinite value, and MS's mobility approaches zero. And this extreme situation is just the case when MS is under service of Geosynchronous Earth Orbit (GEO) satellite.

\subsection{LEO satellite traffic model}

To meet the increasing demand for multimedia services, LEO satellite networks are expected to support real-time interactive multimedia traffic and must be able, therefore, to provide their users with QoS guarantees. The traffic offered to the satellite system is assumed to be the following two classes

- the first is class I real-time multimedia traffic including interactive voice and video applications, such as video on demand $(\mathrm{VoD})$ and video conferencing, and the second is class II nonreal-time data traffic, such as email or ftp. To guarantee its QoS, when a mobile host requests a new connection $C$ in a given cell, it provides the following parameters [9].

- $\quad$ The desire class of traffic for $C$ (either I or II);

- 2) If the request is for a class I connection, the following parameters are also specified: $B_{\text {req }}$ is the required amount of bandwidth for the connection; $\mathrm{B}_{\min }$ is the minimum acceptable amount of bandwidth, that is, the smallest amount of bandwidth that the source requires to maintain acceptable quality, e.g., the smallest encoding rate of its codec.

- $\quad 3$ ) If the request is for a class II connection, only the expected amount of bandwidth $\mathrm{B}_{\min }$ for class II is specified. We assume the mobile hosts are uniformly distributed each cell has independent Poisson new connection.

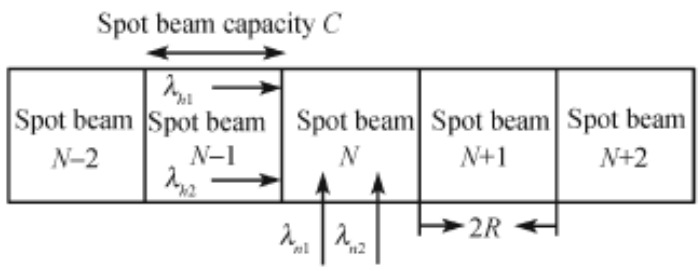

Fig. 2 Traffic model in spot beam $\mathbf{N}$

There are many handover strategies proposed by different scientists. Oliveria C. et al. proposed an adaptive bandwidth reservation scheme [12]. It adjusts the amount of reserved bandwidth based on two parameters: average CDP and reserved bandwidth usage. Different admission strategies are adopted for new connection and handoff connections, and a handoff connection for class I has a higher priority than new connection or class II traffic, so it lowers the CDP for realtime traffic. However, it is found in the simulation that the real-time CDP is still relatively high as class II traffic will occupy bandwidth greedily. In addition, this scheme is designed for terrestrial cellular networks, not taking into account the particular characteristics of LEO satellite's movement. And then it only needs to reserve bandwidth in the next coming cell rather than all the neighbouring cells. 
HUANG et al [16] proposed a new bandwidth allocation strategy named Fairness based channel borrowing strategy in multimedia LEO satellite communication where they have used a channel borrowing database.

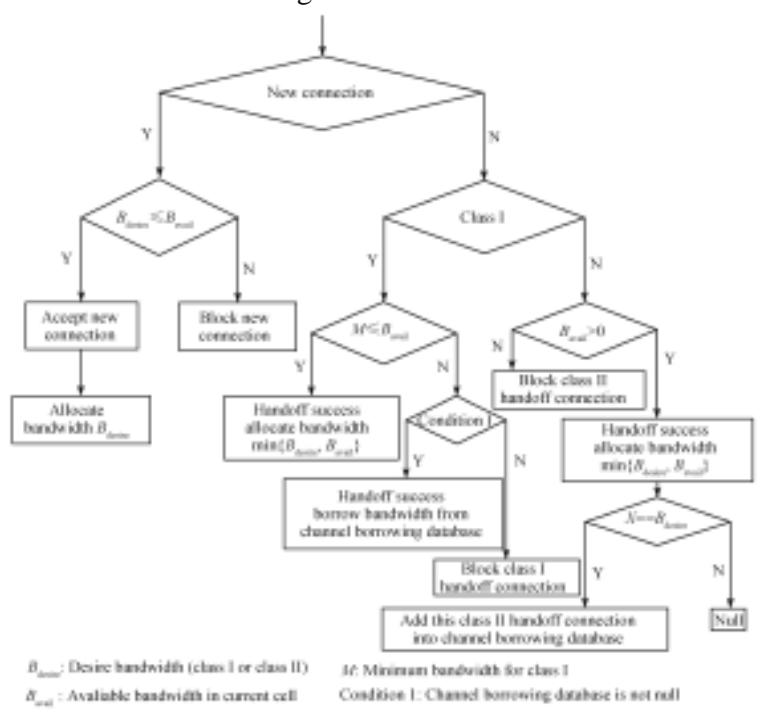

Fig 3 Flowchart for fairness base channel borrowing database strategy

In this strategy a new connection for class I traffic or class II traffic is accepted into the system if the available bandwidth of the current spot beam is greater than its expected bandwidth $M 1, M 2$, respectively, and the expected bandwidth is allocated. Otherwise the new connection is rejected. For a class II handoff connection, if there is available bandwidth in the cell that the connection is moving into, the handoff connection is accepted, and the system allocates enough bandwidth for this handoff connection. If the allocated bandwidth is equal to the expected bandwidth $M 2$ of the class
II traffic, the system adds this connection into the channel borrowing database and starts a timer $T_{\mathrm{i}}$, whose initial value is the entire duration time $T_{\mathrm{c}}$ in one cell? And the function of the timer is to achieve the residual staying time at the current cell. Only if the target cell has no bandwidth does the handoff for the class II traffic fail. For a class I handoff connection, if the amount of available bandwidth in the cell that the connection is moving into is greater than the minimum required bandwidth $M$ of the connection, the handoff connection is accepted, and the system allocates enough bandwidth for this handoff connection. Otherwise the connection enters into the channel borrowing status. If the channel borrowing database is not null (condition 1 in Fig. 3), the system chooses the smallest residual staying time as the channel borrowing object from the channel borrowing database, namely the timer $\mathrm{Ti}$ of the chosen target is equal to $\min \{T i\}$ to reduce the effect of channel borrowing on the class II traffic. If there exists more than one such connection, the system will choose one of them randomly. At the same time, the system reduces bandwidth $M$ from the chosen class II connection to satisfy the minimum required bandwidth $M$ of the class I connection and delete this item from the channel borrowing database. Thus the borrowed bandwidth $M$ is used to support the class I handoff connection. If the channel borrowing database is null, the handoff for the class I traffic fails. Whenever one class II connection in the channel borrowing database finishes its session or has moved out the current cell, the system also deletes its item from the current cell's channel borrowing database. But the main disadvantage of this method is that they have used only a three types of channel borrowing from CBD i.e. MIN, MAX and RAN but not a specific algorithm. They also did not specify what will happen if more than one class I connection wants to handoff that is not specified in their method. In our proposed method we have omitted these disadvantages. 


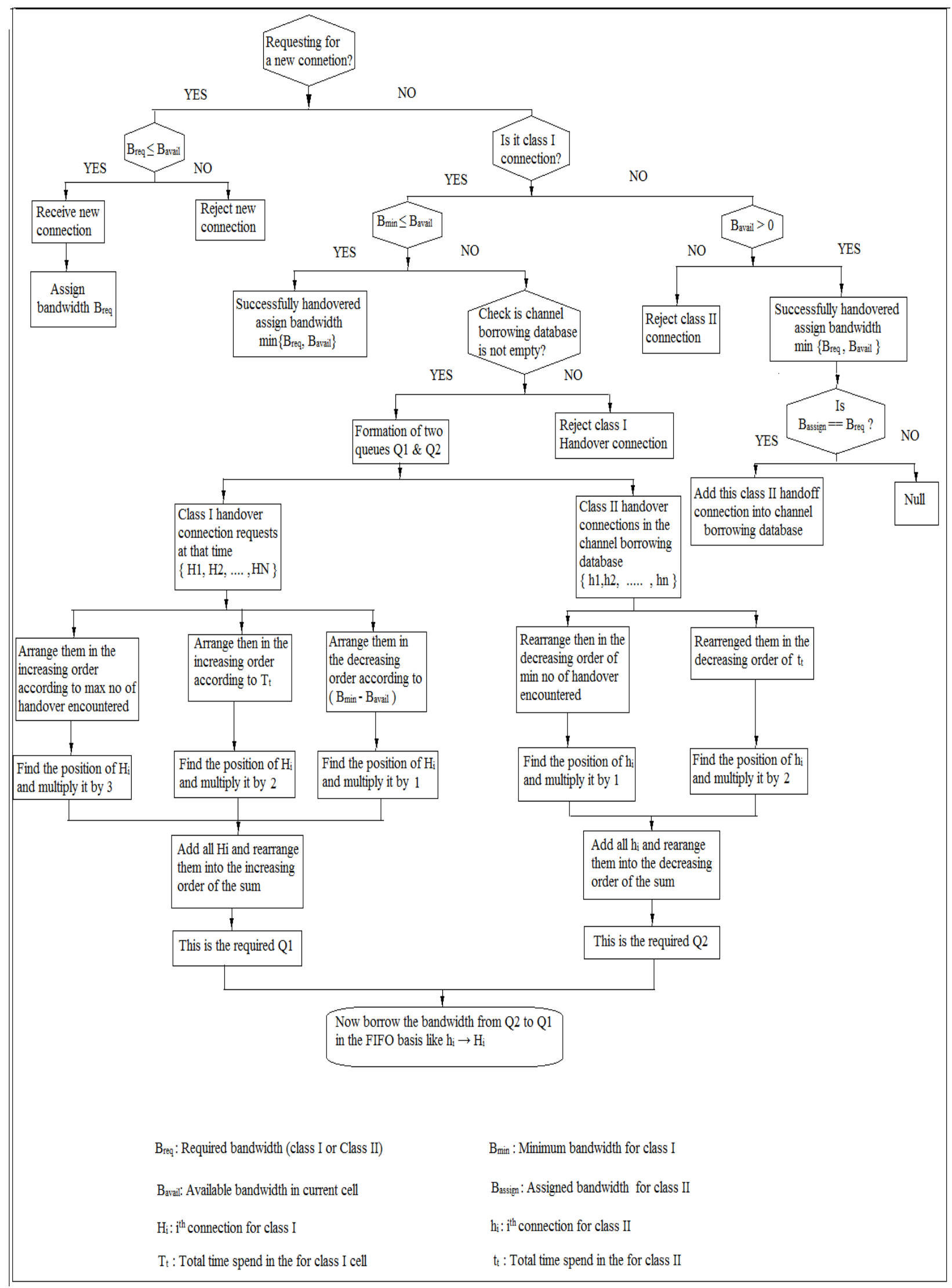

Figure 4: flow chart of CBDA strategy 


\section{PROPOSED WORK}

In our proposed method we introduced a new algorithm named channel borrowing database algorithm (CBDA) strategy for multimedia LEO satellite networks.

Our proposed work is based on the work done by Huang et al [16] where they have used the channel borrowing database but they don't have use and specific algorithm for this i.e. to select class II connection from CBD they just have used Maximum residual time in the cell (MAX), minimum residual time in the sell (MIN), and random selection method (RAN). Here we have used a specific algorithm for CBD and also used two queues Q1 for incoming handover connections class I and Q2 for channel borrowing database class II.

\subsection{Terminologies}

The following terminologies we have used in our algorithm.

$\mathbf{B}_{\text {req }}$ : Required amount of bandwidth for the connection (class I or class II)

$\mathbf{B}_{\min }$ : Minimum amount of bandwidth for the connection of class I

$\mathbf{B}_{\text {avail }}$ : Available amount of bandwidth in the cell

$\mathbf{B}_{\text {assign }}$ : Assigned bandwidth for the connection of class II.

CBDA

Step 1: when a connection requests to join the system it checks whether it is a new connection or not? If it is a new connection then it goes to step 2 else it goes to step 3 .

Step 2: It checks if the required amount of bandwidth for the connection $\mathrm{B}_{\text {req }}$ (both for class I and class II) is less than equal to the available amount of bandwidth $\mathrm{B}_{\text {avail. }}$. If yes then the new connection is admitted into the system and the system assigns the required bandwidth $\mathrm{B}_{\text {req }}$ for the connection else the connection is rejected.

Step 3: In this step the system checks if the connection is class I connection? If yes then it goes to step 4 else it goes to step 5 .

Step 4: Now the CBDA checks if the amount of available bandwidth $\mathrm{B}_{\text {avail }}$ in the cell that the connection is moving into is greater than or equal to the minimum required bandwidth $\mathrm{B}_{\min }$ of the connection, if yes then the handover connection is accepted, and the system assigns minimum bandwidth between $B_{\text {req }}$ (for class $I$ ) and $B_{\text {avail }}$ for this handover connection. Otherwise the connection enters into step 7.

Step 5: when the system determines that the connection is class II connection then it checks if the available amount of bandwidth $\mathrm{B}_{\text {avail }}$ is greater than zero or not. If no then the handover connection is blocked and if yes then the handover is successful and it assigns the minimum bandwidth between $\mathrm{B}_{\text {req }}$ (for class II) and $\mathrm{B}_{\text {avail }}$ and it goes to step 6 .

Step 6: Now the system checks if the assigned amount of bandwidth for class II connection is equal to equal to the required amount of bandwidth $B_{\text {req }}$ ? If it is not the connection is not added to the channel borrowing database. And if yes then this connection is added into the channel borrowing database and starts a timer $T_{i}$, whose initial value is the entire duration time $T_{t}$ in one cell. And the function of the timer is to achieve the residual staying time at the current cell.

Step 7: if the minimum amount of bandwidth is greater than the available amount of bandwidth then the system checks if the channel borrowing database is not empty or not? If it is null then the class I connection is rejected else it goes to step 8.

Step 8: Now the system forms the two queues

$\mathrm{Q} 1=$ the queue for the connection requests for class I at that time

$\mathrm{Q} 2=$ the queue for the class II connections which are admitted in the channel borrowing database. In step 9 we have discussed the rearrangement of Q1 and in step 10 the rearrangement of Q2 is discussed.

Step 9: list of the existing handover connections $\{\mathrm{H} 1$, $\mathrm{H} 2, \ldots, \mathrm{HN}\}$ are arranged on the basis of three conditions where $\mathrm{N}$ is no of existing connection at that time.

$\underline{\mathbf{I}^{\text {st }} \text { condition: }}$ Maximum no of handover encountered by that connection

$2^{\text {nd }}$ condition: The total time spend into the cell $\mathrm{T}_{\mathrm{t}}$

$\underline{3^{\text {rd }} \text { condition: }}$ The extra required bandwidth $\mathrm{B}_{\min }-\mathrm{B}_{\text {avail }}$.

Among them the priority is given to the $1^{\text {st }}$ condition and the system arranged all the connection in an increasing order and multiplies $\mathrm{H}_{\mathrm{i}}$ by 3 where $\mathrm{H}_{\mathrm{i}}$ is the $\mathrm{i}^{\text {th }}$ connection where $\mathrm{i}$ is greater than equal to zero and less than equal to $\mathrm{N}$.

Next the system arranged the condition again in increasing order for the $2^{\text {nd }}$ condition and multiplies $\mathrm{H}_{\mathrm{i}}$ by 2 .

Next the system arranged the $3^{\text {rd }}$ condition in a decreasing order because the minimum required bandwidth will be served first and multiplies $\mathrm{H}_{\mathrm{i}}$ by 1 .

Now the system adds all the $\mathrm{H}_{\mathrm{i}}$ after multiplying and arranged them in the increasing order so that the queue will be served in first in first out FIFO mode. Now the rearrangement of Q1 is complete.

Step 10: The channel borrowing queue Q2 is arranged by the two conditions. Suppose the lists of class II connection in channel borrowing database are $\{\mathrm{h} 1, \mathrm{~h} 2, \ldots \ldots, \mathrm{hn}\}$ where $\mathrm{n}$ is no of class II connection at that time in the database.

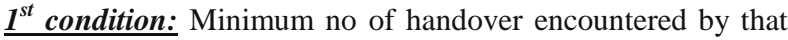
connection

$\underline{2^{\text {nd }} \text { condition: }}$ The total time spend into the cell $\mathrm{t}_{\mathrm{t}}$.

Among the two the least priority is given to condition I as the minimum no of handover occurred by the class II connection will be served first for bandwidth borrowing. So all the connections are arranged in a decreasing order and $h_{i}$ is multiplied by 1 .

But in $2^{\text {nd }}$ condition the connections are arranged in decreasing order as the more serving time connection will be given to more priority. So all the $h_{i}$ are multiplied by 2 .

Now the system adds all the $\mathrm{h}_{\mathrm{i}}$ after multiplying and arranged them in the increasing order so that the queue will be served in first in first out FIFO mode. Now the rearrangement of Q2 is complete.

Step 11: now the system assigns channels form the two queues Q1 and Q2 in FIFO mode like H1 borrows bandwidth from $\mathrm{h} 1, \mathrm{H} 2$ borrows bandwidth from $\mathrm{h} 2$ and continues.

If $\mathrm{N}>\mathrm{n}$ then the $(\mathrm{N}-\mathrm{n})$ connection which don't get and bandwidth are rejected. 
The main advantage of this method that we have used two queues for two types of connection class I and class II and they are rearranged by some specific algorithm.

\section{SIMULATIONS RESULTS}

In order to evaluate the performance of CBDA strategy we compare it to the CBS strategy of MAX, MIN and RAN. Each of them is evaluated by the fairness index, Call dropping probability for class I and class II.

The fairness index is defined by HUANG et al by the formulae

$$
\mathrm{FI}=\frac{\sum \mathrm{AR}(i) \sum \mathrm{AR}(i)}{N \sum[\operatorname{AR}(i) \mathrm{AR}(i)]}
$$

The expression $\operatorname{AR}(i)$ in Eq. (5) is defined as the ratio of the borrowed duration to the connection's whole duration for the $i$ th class II connection in the channel borrowing database. $N$ is the number of class II connections entering the channel borrowing database during the entire simulation. From the definition of the FI, its range is between 0 and 1 , and the bigger the FI is, the more fair the allocation strategy is and the less effect the CBS has on class II connections.

Here in figure 5 we have compared the MAX, MIN , RAN strategy with CBDA strategy and we have found that FI is CBDA has larger FI almost 100\% under different traffic density and MAX has smaller FI. As we have used a specific algorithm so the FI is greater for CBDA strategy

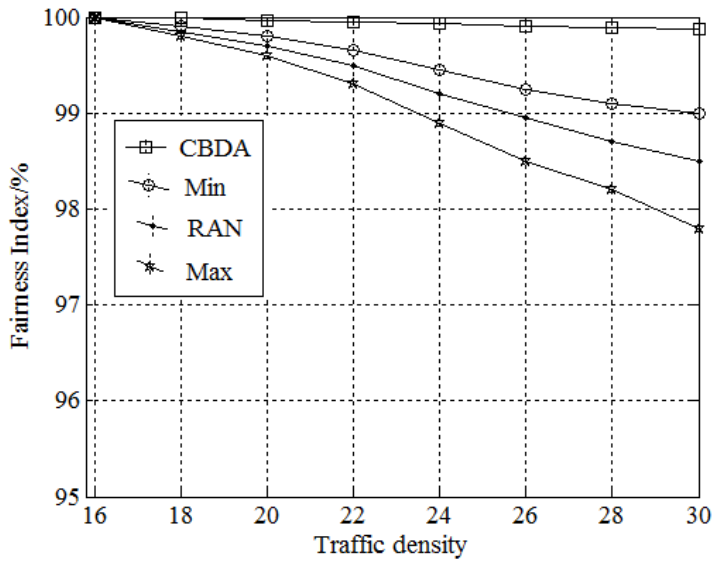

Fig 5 FI index for four channel borrowing strategy

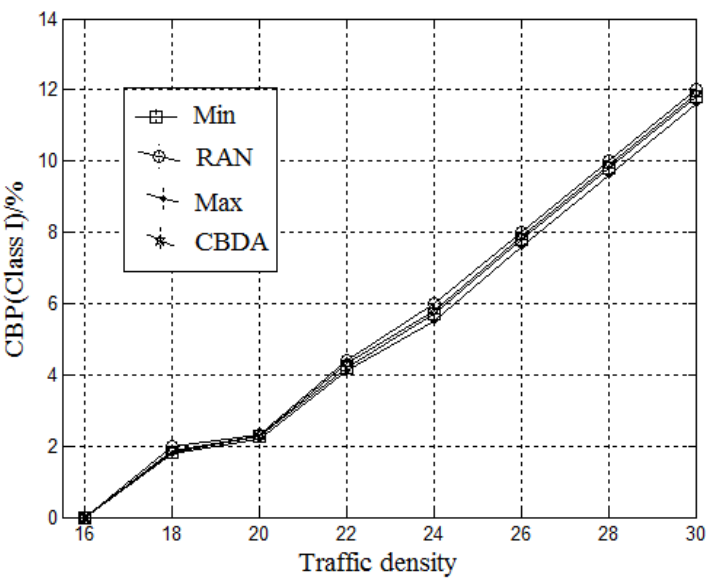

Fig 6 CDP for class I connection

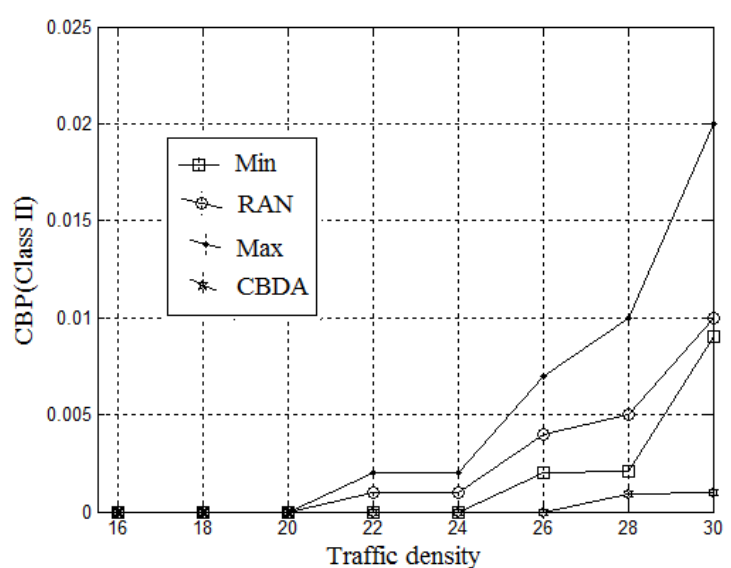

Figure 7 CDP for class II connection

In figure 6 and 7 we have evaluated the Cal dropping probability for both class I and Class II and we have found that the CDP is lower for CBDA method as we have used a specific algorithm for this method.

\section{CONCLUSION}

In this paper a new Channel allocation strategy called Channel borrowing database algorithm (CBDA) has been proposed which reduces the call dropping probability and increases fairness index. Firstly we described different types of satellite systems and details of LEO satellites. Then we described our mobility model and also described the different channel allocation strategies available and mentioned their demerits. Then we described our proposed CBDA strategy and mentioned its advantages. Then we create a virtual environment and we compared the fairness based channel borrowing strategy with MIN, MAX and RAN strategy with our CBDA strategy. Simulation results shows that our CBDA strategy is better than RAN, MAX and MIN strategy.

\section{FUTURE WORK}

In future we will be concentrating on finding better channel allocation strategy to have better call dropping probability.

\section{REFERENCES}

[1] S. L. Kota, P. A. Leppanen, and K. Pahlavan, Broadband Satellite Communications For Internet Access, Kluwer Academic Publishers, 2004.

[2] P. K. Chowdhury, M. Atiquzzaman, W. Ivancic ,Handover Schemes in Satellite Networks: State-of-theart and future research directions IEEE communications surveys 4TH Quarter 2006, Vol. 8, NO. 4A.

[3] Enrico D R, Fantacci R, Giambene G. Characterization of user mobility in low earth orbiting mobile satellite systems. Wireless Networks, 2000, 6(3): 165-179.

[4] Akyldiz I F, Jeong S H. Satellite ATM networks: A survey. IEEE Communications, 1997, 35(7): 30-43.

[5] Maral G, Restrepo J, Enrico D R. Performance analysis for a guaranteed handover service in an LEO constellation with a satellite-fixed cell system. IEEE Transactions on Vehicular Technology, 1998, 47(4): 1 200-1214. 
[6] Debabrata Sarddar, Soumya Das, Dipsikha Ganguli, Sougata Chakraborty, Kunal Hui, Kalyan Kumar Das and Mrinal Kanti Naskar. Article: A New Method for Fast and Low Cost Handover in Leo Satellites. International Journal of Computer Applications 37(7):3945, January 2012. Published by Foundation of Computer Science, New York, USA

[7] Debabrata Sarddar, Soumya Das, Dipsikha Ganguli, Kalyan Kumar Das, Sougata Chakraborty, Kunal Hui and Mrinal Kanti Naskar. Article: A New Method for Controlling Mobility Management Cost of PatHO-LEO satellite and Mobile IP Network. International Journal of Computer Applications 37(7):32-38, January 2012. Published by Foundation of Computer Science, New York, USA.

[8] Todorova P, Olariu S, Nguyen H N. A selective lookahead bandwidth allocation scheme for reliable handoff in multimedia LEO satellite networks. In: The 2nd European Conference on Universal Multiservice Networks. IEEE Press. Colmar, France, 2002, 36-43

[9] El-Kadi M, Olariu S, Wahab H A. A rate-based borrowing scheme for QoS provisioning in multimedia wireless networks. IEEE Transactions on Parallel and Distributed Systems, 2002, 13(2): 156-167

[10] Debabrata Sarddar, Dipsikha Ganguly, Soumya Das, Suman Kumar Sikdar, Sougata Chakraborty, Kunal Hui, Shabnam Bandyopadhyay, Kallyan Kumar Das and Mrinal Kanti Naskar. Article: Introduction of Handover Manager for Better Handover Latency and Handover Throughput during Leo Satellite Handover. International Journal of Computer Applications 42(15):47-52, March 2012. Published by Foundation of Computer Science, New York, USA.

[11] Debabrata Sarddar, Dipsikha Ganguly, Soumya Das, Suman Kumar Sikdar, Sougata Chakraborty, Kunal Hui, Shabnam Bandyopadhyay, Kallyan Kumar Das and Mrinal Kanti Naskar. Article: Area based Mobility Management by using GPS in LEO satellite Networks. International Journal of Computer Applications 42(13):41-43, March 2012. Published by Foundation of Computer Science, New York, USA.

[12] Oliveira C, Kim J B, Suda T. An adaptive bandwidth reservation scheme for high-speed multimedia wireless networks. IEEE Journal on Selected Areas in Communications, 1998, 16(6): 858-874.

[13] Debabrata Sarddar, Soumya Das, Dipsikha Ganguly, Suman Kumar Sikder, Sougata Chakraborty, Kunal Hui, Shabnam Bandyopadhyay, Kalyan Kumar Das and Mrinal Kanti Naskar. Article: Reducing the Mobile IP Binding Updates and the Packet Loss for the Repetitive IP Stations during a Short Period of Time. International Journal of Computer Applications 42(2):27-32, March 2012. Published by Foundation of Computer Science, New York, USA.

[14] Debabrata Sarddar, Soumya Das, Dipsikha Ganguly, Suman Kumar Sikder, Sougata Chakraborty, Kunal Hui, Shabnam Bandyopadhyay, Kalyan Kumar Das and Mrinal Kanti Naskar. Article: A Time based Mobility Management Method for Leo Satellite Networks. International Journal of Computer Applications 42(2):33-40, March 2012. Published by Foundation of Computer Science, New York, USA.
[15] Dipsikha Ganguly, Debabrata Sarddar, Soumya Das, Suman Kumar Sikdar, Sougata Chakraborty and Kunal Hui. Article: Algorithm Based Approach for the Connection Establishment in the Fast Handover in Leo Satellites in BMBHO. International Journal of Computer Applications 44(12):36-42, April 2012. Published by Foundation of Computer Science, New York, USA.

[16] HUANG Fei, XU Hui, WU shiqi, "Fairness basd channel borrowing strategy in multimedia LEO satellite communications", Higher education press and spingerverlag 2007.

[17] Soumya Das, Debabrata Sarddar, Dipsikha Ganguly, Suman Kumar Sikdar, Sougata Chakraborty and Kunal Hui. Article: Location Manager based Handover Method for LEO Satellite Networks. International Journal of Computer Applications44(12):43-49, April 2012. Published by Foundation of Computer Science, New York, USA.

\section{AUTHORS PROFILE}

Debabrata Sarddar is currently pursuing his $\mathrm{PhD}$ at Jadavpur University. He completed his M.Tech in Computer Science \& Engineering from DAVV, Indore in 2006, and his B.Tech in Computer Science \& Engineering from Regional Engineering College, Durgapur in 2001. His research interest includes wireless and mobile communication.

Soumya Das, son of Mr. Subrata Das and Mrs. Swapna Das, currently pursuing his B.Tech in Electronics \& Communication Engg. at Bengal Institute of Technology under West Bengal University of Technology. His research interest includes mobile communication \& satellite communication.

Dipsikha Ganguly, currently pursuing B.Tech in Electronics \& Communication engineering at B.P.Poddar Institute of Management \& Technology under West Bengal University of Technology. Her research interest includes wireless communication \& satellite communication.

Suman Kumar Sikdar completed his B.Tech in CSE from Murshidabad College of Engineering \& Technology and M.Tech in Computer Science \& Engineering from Jadavpur University in 2011. His research interest includes wireless sensor networks and wireless communication systems.

Sougata Chakraborty is presently an employee of IBM (Kolkata). He has completed his B.Tech in IT from Murshidabad College of Engineering and Technology and M.Tech in Computer Science \& Engineering from Jadavpur University in 2011. His research interest includes wireless sensor networks and wireless communication systems.

Kunal Hui is presently an employee of Cognizant (Kolkata). $\mathrm{He}$ has completed his B.Tech in Computer Science \& Engineering from Murshidabad College of Engineering and Technology (2004-2008) and M.Tech in Computer Science \& Engineering from Haldia Institute of Technology (20092011). His research interest includes wireless sensor networks and wireless communication systems.

Sujoy Palit completed his M. Tech degree in Computer Science and Engineering from West Bengal University of Technology in 2005. His research interest includes wireless sensor networks and wireless communication systems. 